[21] S. M. Kay, Fundamentals of Statistical Signal Processing: Estimation Theory. Englewood Cliffs, NJ: Prentice-Hall, 1993, pt. I.

[22] M. Viberg, P. Stoica, and B. Ottersten, "Maximum likelihood array processing in spatially correlated noise fields using parameterized signals," IEEE Trans. Signal Processing, vol. 45, pp. 996-1004, Apr. 1997.

[23] A. Ranheim, "A decoupled approach to adaptive signal separation using an antenna array," IEEE Trans. Veh. Technol., vol. 48, pp. 676-682, May 1999.

[24] N. Seshadri, "Joint data and channel estimation using blind trellis search techniques," IEEE Trans. Commun., vol. 42, pp. 1000-1011, Feb.-Apr 1994.

[25] A. Dogandžić and A. Nehorai, "Finite-length MIMO adaptive equalization using canonical correlation analysis," IEEE Trans. Signal Processing, vol. 50, pp. 984-989, Apr. 2002.

[26] Z. Baranski, A. M. Haimovich, and J. Garcia-Frias, "EM-based iterative receiver for space-time coded modulation with noise variance estimation," in Proc. Globecom Conf., Taipei, Taiwan, R.O.C., Nov. 2002, pp. 355-359.

[27] R. R. Lopes and J. R. Barry, "Exploiting error-control coding in blind channel estimation," in Proc. Globecom Conf., San Antonio, TX, Nov. 2001, pp. 1317-1321.

\section{Robust FIR Filter Design with Envelope Constraints and Channel Uncertainty}

\author{
Ching-Min Lee and I-Kong Fong
}

\begin{abstract}
In this note, a finite impulse response (FIR) filter design problem is considered. The signals to be filtered are assumed to be corrupted by the channel noise. In addition, the channel characteristics are assumed to contain uncertainties. The linear matrix inequalities approach is adopted to provide two optimization procedures for designing $\boldsymbol{H}_{\infty}$ optimal filters and robust $\boldsymbol{H}_{\infty}$ filters subject to filter output envelope constraints. A numerical example is presented to illustrate the proposed filter design methods.
\end{abstract}

Index Terms-Bounded stability, integral quadratic constraints, linear matrix inequality, robust FIR filter, time-domain envelope constraint.

\section{INTRODUCTION}

In the field of signal processing, many filter design problems can be cast as constrained optimization problems. The constraints are usually defined by the specifications of the desired filters, and these specifications arise either from the standards set by certain regulatory bodies or from practical considerations. The time-domain envelope-constrained filter design is one example of these problems, which often involve requirements on the transient responses, such as the pulse-shape requirements in digital data transmission systems. In particular, these kinds of filters may be seen in applications like the pulse compression for many communication and radar systems, the TV waveform equalization with respect to the K-mask, and the data channel equalization or deconvolution, [1]-[3].

As to the optimization part, the $H_{\infty}$ optimization theory has been widely used in robust control and signal processing problems [4]-[8].

Manuscript received Janary 4, 2003; revised July 21, 2003. This work was supported by the National Science Council of the Republic of China under Grant NSC 90-2213-E-002-084. The associate editor coordinating the review of this manuscript and approving it for publication was Dr. Yuan-Pei Lin.

The authors are with the Department of Electrical Engineering, National Taiwan University, Taipei, Taiwan 10617, R.O.C. (e-mail d8921011@ee.ntu.edu.tw; ikfong@cc.ee.ntu.edu.tw).

Digital Object Identifier 10.1109/TSP.2004.827142

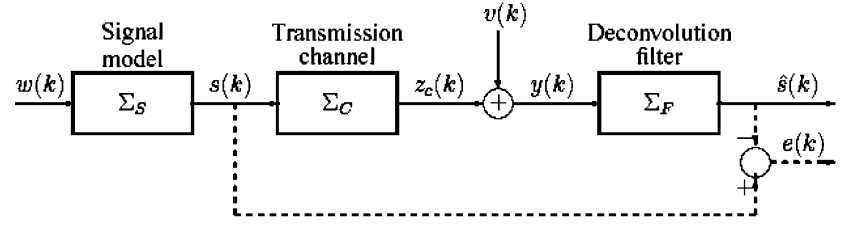

Fig. 1. Deconvolution filtering system.

Take the deconvolution filter design, for example. The objective of the problem may be set to the minimization of the $H_{\infty}$ norm of the filtering error transfer function. In this approach, the system formulation allows the inclusion of the transmission channel and/or signal models. In addition, by utilizing methods developed in [7] and [9], the time-domain envelope constraints may be accommodated simultaneously. However, there are still other important factors to take care of, such as the system uncertainties. In the literature [10], [11], there are some discussions about input uncertainty of the filter, but consideration of input uncertainty bounds only [11] does not fully use information about system uncertainties that may be available. A more direct and complete approach for handling the transmission channel model uncertainties is desirable.

In this correspondence, the $H_{\infty}$ optimal finite impulse response (FIR) filtering problem with envelope constraints and channel uncertainties is studied. The linear matrix inequality (LMI) framework is adopted, and the uncertainties in the channel are formulated as satisfying the integral quadratic constraints (IQCs) [12], [13]. The design method is also enhanced to ensure that the output of the filter is not too close to the constraining envelopes [8], [10], [14]. Compared with some existing method [14], which deals with the output envelope constraint problem by optimization procedures depending on the quasi-Newton method and golden section method, the LMI-based method handling a convex optimization problem is numerically more attractive. To illustrate the effectiveness of the proposed design method, a numerical example is presented.

\section{PROBlem Formulation}

Consider the deconvolution filtering system shown in Fig. 1. In the system, the source signal $s(k) \in \mathcal{R}$ is assumed to be generated by the signal model

$$
\Sigma_{S}:\left\{\begin{array}{l}
x_{s}(k+1)=A_{s} x_{s}(k)+B_{s} w(k) \\
s(k)=C_{s} x_{s}(k)+D_{s} w(k)
\end{array}\right.
$$

where $x_{s}(k) \in \mathcal{R}^{n_{s}}$ is the model state vector, $w(k) \in l_{2}[0, \infty)$ is the driving signal of the model, and $A_{s}, B_{s}, C_{s}$, and $D_{s}$ are known constant matrices of appropriate dimensions. The source signal is transmitted through a channel with an uncertain characteristic modeled by

$$
\Sigma_{C}:\left\{\begin{array}{l}
x_{c}(k+1)=A_{c} x_{c}(k)+B_{c} s(k)+\sum_{i=1}^{p} H_{c 1 i} \xi_{c i}(k) \\
z_{c}(k)=C_{c} x_{c}(k)+D_{c} s(k)+\sum_{i=1}^{p} H_{c 2 i} \xi_{c i}(k)
\end{array}\right.
$$

where $x_{c}(k) \in \mathcal{R}^{n_{c}}$ is the channel state vector, $\xi_{c i}(k) \in \mathcal{R}^{n_{i}}, i=$ $1,2, \ldots, p$ is the $i$ th uncertain vector satisfying the IQC [12]

$$
\sum_{k=0}^{\mu}\left\|\xi_{c i}(k)\right\|^{2} \leq \sum_{k=0}^{\mu}\left\|E_{1 i} x_{c}(k)+E_{2 i} s(k)+E_{3 i} \xi_{c}(k)\right\|^{2}
$$

as $\mu \rightarrow \infty, \xi_{c}^{T}(k)=\left[\begin{array}{lll}\xi_{c 1}^{T}(k) & \cdots & \xi_{c p}^{T}(k)\end{array}\right]$, and $A_{c}, B_{c}, C_{c}$, $D_{c}, H_{c 1 i}, H_{c 2 i}, E_{1 i}, E_{2 i}$, and $E_{3 i}$ are known constant matrices with appropriate dimensions. For subsequent usage, we define $H_{c 1}=\left[\begin{array}{lll}H_{c 11} & \cdots & H_{c 1 p}\end{array}\right], H_{c 2}=\left[\begin{array}{lll}H_{c 21} & \cdots & H_{c 2 p}\end{array}\right]$, $E_{1}^{T}=\left[\begin{array}{lll}E_{11}^{T} & \cdots & E_{1 p}^{T}\end{array}\right], E_{2}^{T}=\left[\begin{array}{lll}E_{21}^{T} & \cdots & E_{2 p}^{T}\end{array}\right]$, and $E_{3}^{T}=$ $\left[\begin{array}{lll}E_{31}^{T} & \cdots & E_{3 p}^{T}\end{array}\right]$. 
At the receiving end, the measured signal $y(k)$ is equal to $z_{c}(k)+$ $v(k)$, where $v(k)$ is the energy-bounded channel noise. To optimally recover the source signal $s(k)$, the signal $y(k)$ is deconvoluted by a filter

$$
\Sigma_{F}:\left\{\begin{array}{l}
x_{f}(k+1)=A_{f} x_{f}(k)+B_{f} y(k) \\
\hat{s}(k)=C_{f} x_{f}(k)+D_{f} y(k)
\end{array}\right.
$$

where $x_{f}(k) \in \mathcal{R}^{n_{f}}$ is the filter state vector, and $A_{f}, B_{f}, C_{f}$, and $D_{f}$ are constant matrices to be designed. Let the filtering error be defined as $e(k)=s(k)-\hat{s}(k)$. Then, it satisfies

$$
\Sigma_{e}:\left\{\begin{array}{l}
x_{e}(k+1)=A_{e} x_{e}(k)+B_{e} w_{e}(k)+H_{1 e} \xi_{c}(k) \\
e(k)=C_{e} x_{e}(k)+D_{e} w_{e}(k)+H_{2 e} \xi_{c}(k)
\end{array}\right.
$$

where $x_{e}^{T}(k)=\left[x_{s}^{T}(k) x_{c}^{T}(k) x_{f}^{T}(k)\right], w_{e}^{T}(k)=\left[w^{T}(k) v^{T}(k)\right]$, and

$$
\begin{aligned}
A_{e} & =\left[\begin{array}{ccc}
A_{s} & 0 & 0 \\
B_{c} C_{s} & A_{c} & 0 \\
B_{f} D_{c} C_{s} & B_{f} C_{c} & A_{f}
\end{array}\right] \\
B_{e} & =\left[\begin{array}{cc}
B_{s} & 0 \\
B_{c} D_{s} & 0 \\
B_{f} D_{c} D_{s} & B_{f}
\end{array}\right], H_{1 e}=\left[\begin{array}{c}
0 \\
H_{c 1} \\
B_{f} H_{c 2}
\end{array}\right] \\
C_{e} & =\left[\begin{array}{lll}
C_{s}-D_{f} D_{c} C_{s}-D_{f} C_{c}-C_{f}
\end{array}\right], \\
D_{e} & =\left[\begin{array}{lll}
D_{s}-D_{f} D_{c} D_{s}-D_{f}
\end{array}\right], H_{2 e}=-D_{f} H_{c 2} .
\end{aligned}
$$

The purpose of this paper is to design an FIR filter $\Sigma_{F}$ with three desired properties, of which the first one is given by the following definition.

Definition 1: [12] The filtering error dynamics $\Sigma_{e}$ is called bounded stable if there exists a constant $\beta \geq 0$ such that $\left\|x_{e}(k)\right\| \leq \beta$ for all $k \geq 0$, no matter what the initial condition $x_{e}(0)$ and input $w_{e}(k) \in$ $l_{2}[0, \infty)$ are.

The second desired property of the filter is that for a given filter input signal $y(k)$ of finite duration, the output signal $\hat{s}(k)$ has to be bounded above and below by

$$
l_{s}(k) \leq \hat{s}(k) \leq u_{s}(k)
$$

for $k=0,1, \ldots, n$, where $l_{s}(k)$ and $u_{s}(k)$ are the lower and upper bounds of the time-domain mask, respectively, and $n+1$ is the duration of $\hat{s}(k)$. Finally, it is desired that when $x_{e}(0)=0$, the filter has the $H_{\infty}$ performance

$$
\gamma^{-1} \sum_{k=0}^{\mu}\|e(k)\|^{2} \leq \gamma \sum_{k=0}^{\mu}\left\|w_{e}(k)\right\|^{2}
$$

for some scalar $\gamma>0$ and all $w_{e} \neq 0$, as $\mu \rightarrow \infty$.

\section{FIR FILTER DESIGN}

To start, the $\mathcal{S}$-procedure [15] is applied to convert two of the above design objectives into a quadratic condition, and a theorem is derived.

Theorem 1: Under all admissible channel uncertainties satisfying the IQC (3), the error dynamics $\Sigma_{e}$ is bounded stable and satisfies (8) when $x_{e}(0)=0$ if there exists a symmetric positive definite matrix $X \in \mathcal{R}^{n_{s}+n_{c}+n_{f}}$ and positive scaling scalars $\tau_{1}, \tau_{2}, \ldots, \tau_{p}, \gamma$ satisfying the following inequality:

$$
\begin{aligned}
& \left(A_{e} x_{e}+B_{e} w_{e}+H_{1 e} \xi_{c}\right)^{T} X\left(A_{e} x_{e}+B_{e} w_{e}+H_{1 e} \xi_{c}\right) \\
& \quad-x_{e}^{T} X x_{e}+\sum_{i=1}^{p} \tau_{i}\left(\left\|E_{1 i} x_{c}+E_{2 i} s+E_{3 i} \xi_{c}\right\|^{2}-\left\|\xi_{c_{i}}\right\|^{2}\right) \\
& +\gamma^{-1}\left\|C_{e} x_{e}+D_{e} w_{e}+H_{2 e} \xi_{c}\right\|^{2}-\gamma\left\|w_{e}\right\|^{2}<0
\end{aligned}
$$

Proof: Summing up the left-hand side of the inequality in (9) along any trajectory of the error dynamics $\Sigma_{e}$, one gets

$$
\begin{aligned}
& x_{e}^{T}(\mu+1) X x_{e}(\mu+1)-x_{e}^{T}(0) X x_{e}(0) \\
& +\sum_{i=1}^{p} \tau_{i}\left\{\sum_{k=0}^{\mu}\left\|E_{1 i} x_{c}(k)+E_{2 i} s(k)+E_{3 i} \xi_{c}(k)\right\|^{2}\right. \\
& \left.\quad-\sum_{k=0}^{\mu}\left\|\xi_{c i}(k)\right\|^{2}\right\} \\
& +\left\{\gamma^{-1} \sum_{k=0}^{\mu}\|e(k)\|^{2}-\gamma \sum_{k=0}^{\mu}\left\|w_{e}(k)\right\|^{2}\right\}<0
\end{aligned}
$$

for all $w_{e} \neq 0$. From (3), it follows that

$$
x_{e}^{T}(\mu+1) X x_{e}(\mu+1)<x_{e}^{T}(0) X x_{e}(0)+\gamma \sum_{k=0}^{\mu}\left\|w_{e}(k)\right\|^{2}
$$

as $\mu \rightarrow \infty$. Thus, the bounded stability of the filtering error dynamics is implied. As to the $H_{\infty}$ performance, when $x_{e}(0)=0$, it follows from (3) and (10) that (8) holds.

\section{A. $H_{\infty}$ Optimal FIR Filter Design}

To develop a set of easy-to-use conditions for designing the FIR filters, the parameter matrices of the filter $\Sigma_{F}$ are assumed to be of the form [5]

$$
\begin{aligned}
& A_{f}=\left[\begin{array}{ccccc}
0 & 1 & 0 & \cdots & 0 \\
0 & 0 & 1 & \cdots & 0 \\
\vdots & \vdots & \vdots & \ddots & \vdots \\
0 & 0 & 0 & \cdots & 1 \\
0 & 0 & 0 & \cdots & 0
\end{array}\right]_{n_{f} \times n_{f}} \quad, \quad B_{f}=\left[\begin{array}{c}
0 \\
0 \\
\vdots \\
0 \\
1
\end{array}\right]_{n_{f} \times 1} \\
& C_{f}=\left[\begin{array}{llll}
f\left(n_{f}\right) & f\left(n_{f-1}\right) & \cdots & f(1)
\end{array}\right], \quad D_{f}=f(0)
\end{aligned}
$$

where $f(0), f(1), \cdots, f\left(n_{f}\right)$ are the parameters to be determined. Accordingly, the transfer function of the filter is

$$
\begin{aligned}
C_{f}\left(z I-A_{f}\right)^{-1} B_{f}+D_{f} \\
\quad=f(0)+f(1) z^{-1}+f(2) z^{-2}+\cdots+f\left(n_{f}\right) z^{-n_{f}} .
\end{aligned}
$$

At this point, it is appropriate to consider the second desired property of the filter. Suppose that a signal $\{y(0), y(1), y(2), \ldots, y(m), 0,0, \ldots\}$ is given, and it is required that (7) be satisfied. Let [7]

$$
y=\left[\begin{array}{c}
y(0) \\
y(1) \\
\vdots \\
y(m)
\end{array}\right], f=\left[\begin{array}{c}
f(0) \\
f(1) \\
\vdots \\
f\left(n_{f}\right)
\end{array}\right], l_{b}=\left[\begin{array}{c}
l_{s}(0) \\
l_{s}(1) \\
\vdots \\
l_{s}(n)
\end{array}\right], u_{b}=\left[\begin{array}{c}
u_{s}(0) \\
u_{s}(1) \\
\vdots \\
u_{s}(n)
\end{array}\right]
$$

and

$$
Y=\left[\begin{array}{cccc}
y(0) & 0 & \cdots & 0 \\
y(1) & y(0) & \cdots & 0 \\
\vdots & y(1) & \cdots & \vdots \\
y(m) & \vdots & & y(0) \\
0 & y(m) & \vdots & y(1) \\
\vdots & \vdots & & \vdots \\
0 & 0 & \cdots & y(m)
\end{array}\right]
$$

where $Y$ is an $n \times\left(n_{f}+1\right)$ matrix, and $n=m+n_{f}+1$. Therefore, the time-domain constraints in (7) are equivalent to

$$
\operatorname{diag}\left(l_{b}\right) \leq \operatorname{diag}(Y f) \leq \operatorname{diag}\left(u_{b}\right)
$$


where "diag $(\cdot)$ " denotes a diagonal matrix formed by putting the elements of the argument vector on the diagonal positions of the matrix. Based on the inequality (14) and Theorem 1, the following theorem can be established to provide a convex optimization problem with the LMI formulation, which may be easily solved for finding the filter parameters.

Theorem 2: An $H_{\infty}$ optimal FIR filter of the form (11) satisfying the three desired properties formulated in Section II exists if the following optimization problem has solutions:

$$
\min _{X, f, J} \gamma
$$

subject to (16), shown at bottom of the page, and

$$
\begin{aligned}
& \operatorname{diag}\left(l_{b}\right) \leq \operatorname{diag}(Y f) \\
& \operatorname{diag}(Y f) \leq \operatorname{diag}\left(u_{b}\right) \\
& X>0, J>0, \quad \gamma>0
\end{aligned}
$$

where $J=\operatorname{diag}\left(\tau_{1} I_{n_{1}}, \cdots, \tau_{p} I_{n_{p}}\right), C_{e}=\left[\begin{array}{lll}C_{s} & 0 & 0\end{array}\right]-D_{f}$ $\left[\begin{array}{lll}D_{c} C_{s} & C_{c} & 0\end{array}\right]-C_{f}\left[\begin{array}{lll}0 & 0 & I\end{array}\right], D_{e}=\left[\begin{array}{ll}D_{s} & 0\end{array}\right]-D_{f}\left[\begin{array}{ll}D_{c} D_{s} & I\end{array}\right]$, $E_{e}=\left[\begin{array}{lll}E_{2} C_{s} & E_{1} & 0\end{array}\right]$, and $E_{w}=\left[\begin{array}{ll}E_{2} D_{s} & 0\end{array}\right]$.

Proof: Note that (9) can be rewritten as

$$
\begin{aligned}
& \left(A_{e} x_{e}+B_{e} w_{e}+H_{1 e} \xi_{c}\right)^{T} X\left(A_{e} x_{e}+B_{e} w_{e}+H_{1 e} \xi_{c}\right)-x_{e}^{T} X x_{e} \\
& \quad+\left(E_{e} x_{e}+E_{w} w_{e}+E_{3} \xi_{c}\right)^{T} J\left(E_{e} x_{e}+E_{w} w_{e}+E_{3} \xi_{c}\right) \\
& -\xi_{c}^{T} J \xi_{c}+\gamma^{-1}\left(C_{e} x_{e}+D_{e} w_{e}+H_{2 e} \xi_{c}\right)^{T} \\
& \times\left(C_{e} x_{e}+D_{e} w_{e}+H_{2 e} \xi_{c}\right)-\gamma w_{e}^{T} w_{e} \\
& \quad=\left[\begin{array}{lll}
x_{e}^{T} & w_{e}^{T} & \xi_{c}^{T}
\end{array}\right] M\left[\begin{array}{lll}
x_{e}^{T} & w_{e}^{T} & \xi_{c}^{T}
\end{array}\right]^{T}<0 .
\end{aligned}
$$

\section{B. Robust $H_{\infty}$ FIR Filter Design}

When an $H_{\infty}$ optimal FIR filter is designed using Theorem 2 in the above subsection, the time-domain constraints in (7) will be satisfied. However, in some cases, the output response of the filter may be very close to or even touch the constraint envelopes. In certain applications, this is regarded as undesired. To avoid this kind of situation, the envelope constraints and objective function considered in Theorem 2 can be modified as

$$
m_{s}(k)-\eta \cdot e_{s}(k) \leq \hat{s}(k) \leq m_{s}(k)+\eta \cdot e_{s}(k)
$$

for $k=0,1, \ldots, n$, and

$$
\min _{X, f, J}\left(\rho_{1} \gamma+\rho_{2} \eta\right)
$$

respectively, where

$$
\begin{aligned}
m_{s}(k) & =\frac{1}{2}\left(u_{s}(k)+l_{s}(k)\right) \\
e_{s}(k) & =\frac{1}{2}\left(u_{s}(k)-l_{s}(k)\right)
\end{aligned}
$$

$\rho_{1}$ and $\rho_{2}$ are tunable weighting parameters, and $0<\eta<1$ is a new scaling parameter to be determined. Note that (20) is equivalent to the LMIs

$$
\operatorname{diag}\left(m_{b}\right)-\eta \cdot \operatorname{diag}\left(e_{b}\right) \leq \operatorname{diag}(Y f)
$$

and

$$
\operatorname{diag}(Y f) \leq \operatorname{diag}\left(m_{b}\right)+\eta \cdot \operatorname{diag}\left(e_{b}\right)
$$

where

$$
m_{b}=\left[\begin{array}{c}
m_{s}(0) \\
m_{s}(1) \\
\vdots \\
m_{s}(n)
\end{array}\right], \quad e_{b}=\left[\begin{array}{c}
e_{s}(0) \\
e_{s}(1) \\
\vdots \\
e_{s}(n)
\end{array}\right]
$$

and $f$ and $Y$ are defined in (12) and (13).

\section{NUMERICAL EXAMPLE}

In this section, an example is worked out to illustrate the proposed design algorithms. Suppose that the system shown in Fig. 1 has the signal model $\Sigma_{S}$ with the following system matrices:

$$
\begin{aligned}
& A_{s}^{T}=\left[\begin{array}{llllll}
-2.3060 & 1 & 0 & 0 & 0 & 0 \\
-2.9625 & 0 & 1 & 0 & 0 & 0 \\
-2.2590 & 0 & 0 & 1 & 0 & 0 \\
-1.0922 & 0 & 0 & 0 & 1 & 0 \\
-0.3009 & 0 & 0 & 0 & 0 & 1 \\
-0.0325 & 0 & 0 & 0 & 0 & 0
\end{array}\right], \quad B_{s}=\left[\begin{array}{l}
1 \\
0 \\
0 \\
0 \\
0 \\
0
\end{array}\right] \\
& C_{s}=\left[\begin{array}{llllll}
0 & 0 & 0 & 0 & 0.0062 & 0.2170
\end{array}\right], \quad D_{s}=0
\end{aligned}
$$

and the channel model $\Sigma_{C}$ with the following system matrices:

$$
\begin{aligned}
& A_{c}=\left[\begin{array}{ccc}
-1.90 & -1.18 & -0.24 \\
1 & 0 & 0 \\
0 & 1 & 0
\end{array}\right], \quad B_{c}=\left[\begin{array}{l}
1 \\
0 \\
0
\end{array}\right] \\
& C_{c}=\left[\begin{array}{lll}
-0.360 & -0.153 & -0.027
\end{array}\right], \quad D_{c}=0.45 .
\end{aligned}
$$

To simplify the discussion, assume that there is only one uncertain variable in the channel. The related matrix parameters are $H_{c 1}^{T}=H_{c 11}^{T}=\left[\begin{array}{lll}0.02 & 0.03 & 0.01\end{array}\right], H_{c 2}=H_{c 21}=0.024$, $E_{1}=E_{11}=\left[\begin{array}{lll}0.62 & 0.51 & 0.55\end{array}\right], E_{2}=E_{21}=0.73$, and $E_{3}=E_{31}=-0.082$. In addition, assume, to start, that there is no channel noise (i.e., $v \equiv 0$ ). In Fig. 2, the filter output constraint envelopes corresponding to a particular channel output $y(k)$ are shown. This $y(k)$ is generated by the unit-impulse response of the signal model when the uncertain variable of the channel model equals zero. Then, the $H_{\infty}$ optimal filter is designed using the LMI Toolbox of Matlab [16] with $n_{f}$ selected to be 15 . The resultant optimal $\gamma=3.5330$, and the filter output with respect to the above $y(k)$ is also displayed in Fig. 2. Clearly, the output is quite close to the lower envelope. Thus, the robust $H_{\infty}$ filter is designed with $\rho_{1}=1$ and $\rho_{2}=1.5$. The resultant optimal $\gamma$ and $\eta$ are 3.8891 and 0.6243, respectively. In Fig. 3, the new filter output is displayed, which is very close to the envelope center line, as desired.

To see the robustness of the two filters, the filter outputs are computed again with respect to the same signal $s(k)$, but this time, a normally distributed white channel noise $v(k)$ with zero mean and variance equal to $1 \times 10^{-4}$ is added. In addition, the channel uncertain variable $\xi_{c 1}(k)$ is set to be $-3.356 k /\left(k^{2}+k+1\right)$ for $k \geq 0$. The results are shown in Figs. 4 and 5. It is seen that most of the time, the filter

$$
M=\left[\begin{array}{llll}
A_{e}^{T} X A_{e}-X+E_{e}^{T} J E_{e} & A_{e}^{T} X B_{e}+E_{e}^{T} J E_{w} & A_{e}^{T} X H_{1 e}+E_{e}^{T} J E_{3} & C_{e}^{T} \\
B_{e}^{T} X A_{e}+E_{w}^{T} J E_{e} & B_{e}^{T} X B_{e}+E_{w}^{T} X E_{w}-\gamma I & B_{e}^{T} X H_{1 e}+E_{w}^{T} J E_{3} & D_{e}^{T} \\
H_{1 e}^{T} X A_{e}+E_{3}^{T} J E_{e} & H_{1 e}^{T} X B_{e}+E_{3}^{T} J E_{w} & H_{1 e}^{T} X H_{1 e}+E_{3}^{T} J E_{3}-J & H_{2 e}^{T} \\
C_{e} & D_{e} & H_{2 e} & -\gamma I
\end{array}\right]<0
$$




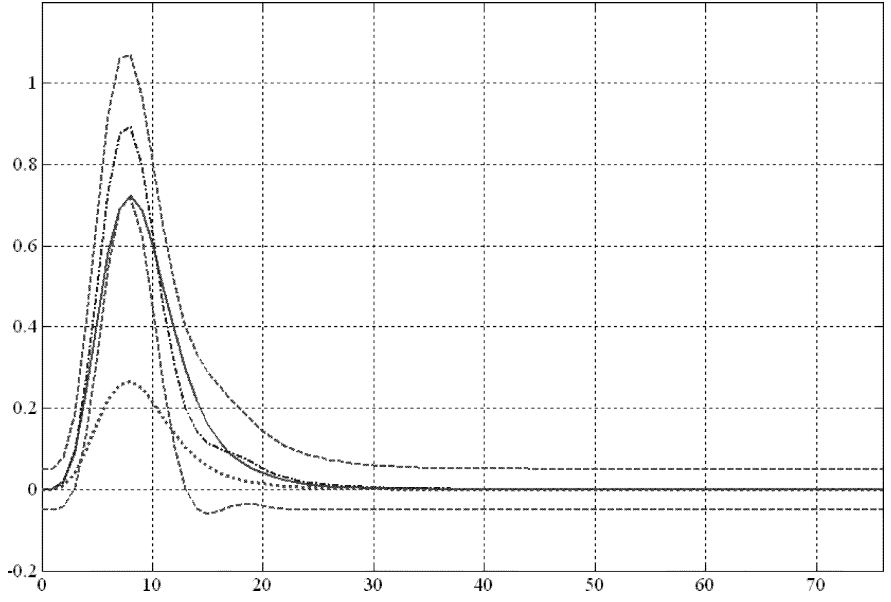

Fig. 2. Filter output $\boldsymbol{y}(\boldsymbol{k})$ (dotted line), the corresponding filter output constraint envelopes (dashed lines), and output of the $\boldsymbol{H}_{\infty}$ optimal filter (solid line).

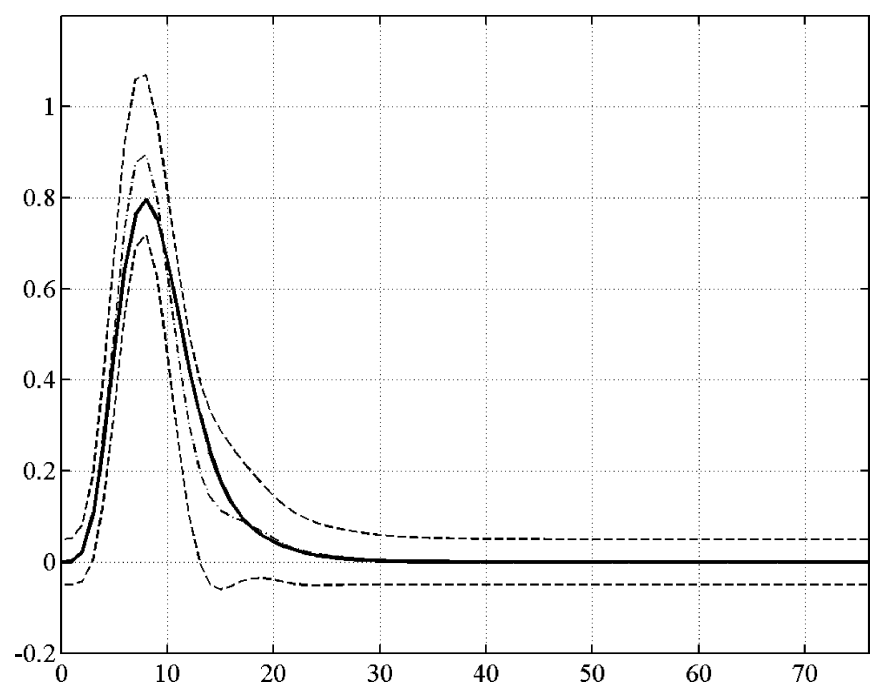

Fig. 3. Center line of the mask (dash-dotted line) and output of the robust $\boldsymbol{H}_{\infty}$ filter (solid line).

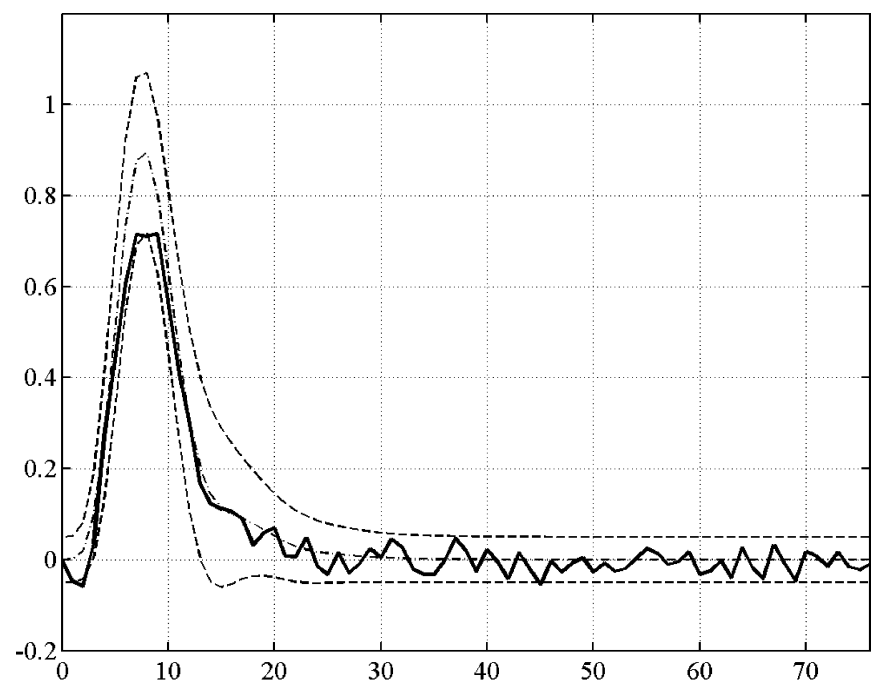

Fig. 4. Filter output of the $\boldsymbol{H}_{\infty}$ optimal filter (solid line) with channel noise and uncertainty.

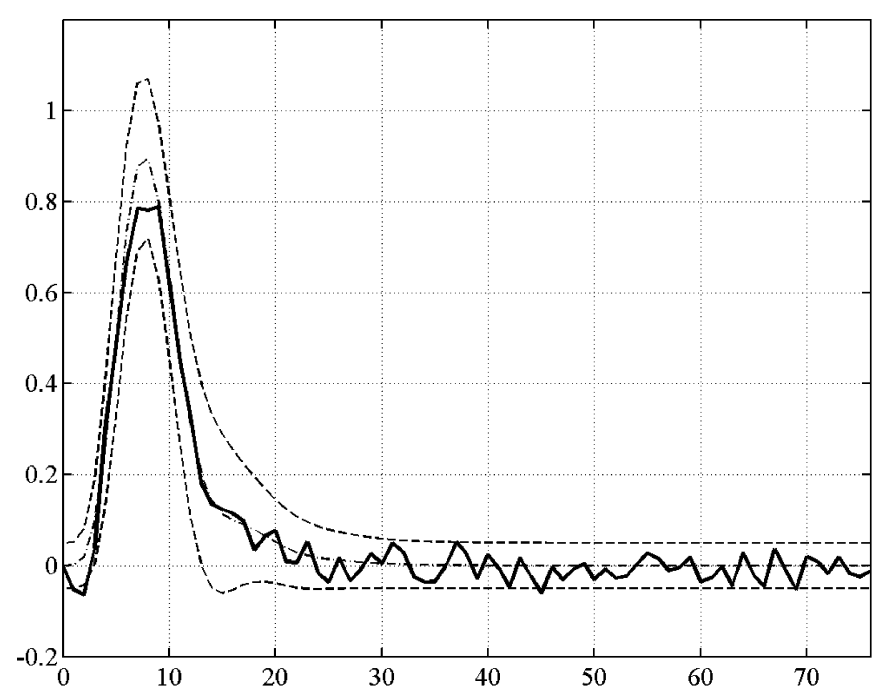

Fig. 5. Filter output of the robust $\boldsymbol{H}_{\infty}$ filter (solid line) with channel noise and uncertainty.

output of the robust $H_{\infty}$ filter is kept within the envelopes, whereas the situation for the optimal $H_{\infty}$ filter is relatively worse.

\section{DISCUSSION AND CONCLUSION}

In this correspondence, an LMI-based optimization approach is proposed to design FIR filters that satisfy prespecified time-domain envelope constraints at the output. In addition to the widely treated channel noise, the less-considered channel uncertainties are accommodated using the versatile IQC formulation. Both the $H_{\infty}$ optimal filter and the robust $H_{\infty}$ filter are offered so that the tradeoff between the $H_{\infty}$ performance and constraint robustness may be carried out.

\section{REFERENCES}

[1] R. J. Evans, T. E. Eortmann, and A. Cantoni, "Envelope-constrained filters-I: theory and applications," IEEE Trans. Inform. Theory, vol. IT-23, pp. 421-434, July 1977.

[2] B. Vo, A. Cantoni, and K. L. Teo, "Envelope constrained filter with linear interpolator," IEEE Trans. Signal Processing, vol. 45, pp. 1405-1414, June 1997.

[3] — Filter Design With Time Domain Mask Constraints: Theory and Applications. Dordrecht, The Netherlands: Kluwer, 2001.

[4] A. Sideris and H. Rotstein, "Single-input-single-output $\boldsymbol{H}_{\infty}$ control with time domain constraints," Automatica, vol. 29, pp. 969-983, July 1993.

[5] H. Rotstein and A. Sideris, " $\boldsymbol{H}_{\infty}$ optimization with time domain constraints," IEEE Trans. Automat. Contr., vol. 39, pp. 762-779, Apr. 1994.

[6] M. J. Grimble, " $\boldsymbol{H}_{\infty}$ inferential filtering, prediction and smoothing problems," Signal Process., vol. 60, pp. 289-304, Aug. 1997.

[7] Z. Tan, Y. C. Soh, and L. Xie, "Envelope-constrained $\boldsymbol{H}_{\infty}$ FIR filter design,” IEEE Trans. Circuits Syst. II, vol. 47, pp. 79-82, Jan. 2000.

[8] Z. Zang, A. Cantoni, and K. L. Teo, "Envelope-constrained IIR filter design via $\boldsymbol{H}_{\infty}$ optimization methods," IEEE Trans. Circuits Syst., vol. 46, pp. 649-653, June 1999.

[9] Z. Tan, Y. C. Soh, and L. Xie, "Envelope-constrained $\boldsymbol{H}_{\infty}$ filter design: an LMI optimization approach," IEEE Trans. Signal Processing, vol. 48, pp. $2960-2963$, Oct. 2000.

[10] B. Vo and A. Cantoni, "Continuous-time envelope constrained filter design with input uncertainty," Trans. Circuits Syst. I, vol. 47, pp. 1445-1454, Oct. 2000.

[11] W. X. Zheng, A. Cantoni, and K. L. Teo, "Sensitivity of envelope-constrained filters with uncertain input," IEEE Trans. Circuits Syst., vol. 42, pp. 509-516, Sept. 1995.

[12] H. Li and M. Fu, "A linear matrix inequality approach to robust $\boldsymbol{H}_{\infty}$ filter," IEEE Trans. Signal Processing, vol. 45, pp. 2338-2350, Sept. 1997. 
[13] L. Xie, M. Fu, and H. Li, "Passivity analysis and passification for uncertain signal processing systems," IEEE Trans. Signal Processing, vol. 46, pp. 2394-2403, Sept. 1998.

[14] C. H. Tseng, K. L. Teo, A. Cantoni, and Z. Zang, "Design of robust continuous-time filter design with orthonormal bases," IEEE Trans. Signal Processing, vol. 48, pp. 2881-2891, Oct. 2000.

[15] S. Boyd, L. E. Ghaoui, E. Feron, and V. Balakrishnan, Linear Matrix Inequalities in System and Control Theory. Philadelphia, PA: SIAM, 1994.

[16] P. Gahinet, A. Nemirovski, A. J. Laub, and M. Chilali, LMI Control Toolbox-for Use With MATLAB. Natick, MA: The Math Works Inc., 1995.

\section{Eigenstructure Approach for Complete Characterization of Linear-Phase FIR Perfect Reconstruction Analysis Length $2 M$ Filterbanks}

Anamitra Makur, Arigovindan Muthuvel, and P. Viswanadha Reddy

\begin{abstract}
The eigenstructure based characterization of $M$-channel finite impulse response perfect reconstruction (FIR PR) filterbanks in a previous paper by the authors is extended here to the linear-phase case. Some results relating to linear-phase filterbanks is derived by finding appropriate restrictions on the eigenstructure of the analysis polyphase matrix. Consequently, a complete and minimal characterization for such filterbanks with all analysis length $2 M$ and any synthesis length is developed. Parameterization and design examples are also presented.
\end{abstract}

Index Terms-Eigenstructure, FIR filterbank, linear phase.

\section{INTRODUCTION}

In [1], we used the eigenstructure representation of the polyphase matrix to propose complete characterizations of finite impulse response perfect reconstruction (FIR PR) $M$-channel filterbanks with first-order analysis polyphase matrix. Linear-phase FIR perfect reconstruction filterbanks (LPFBs) find application in many signal and image processing fields. In this correspondence, we extend the eigenstructure representation to obtain a complete characterization of linear-phase FIR perfect reconstruction $M$-channel filterbanks with all analysis filter lengths being $2 M$ (hence first-order analysis polyphase matrix), which are referred to henceforth as FOLPFBs. The synthesis filter length in this characterization is not restricted to $2 M$ as is conventionally done but may take a value up to $M^{2}$

Characterization of a subclass of LPFBs, such as orthogonal, $M=2$, or $M=3$, has been reported on several occasions. The design of FOLPFBs with a multistage structure using the discrete cosine transform (DCT) such that a fast implementation exists is reported in [2], where it is called the lapped biorthogonal transform. Design of an LPFB of any order is reported in [3], when $K$ filters are given such that the part polyphase matrix has rank $K$ for all $z^{-1}$ except $z^{-1}=0$,

Manuscript received July 9, 2002; revised July 7, 2003. This work was done at the Electrical Communication Engineering Department, Indian Institute of Science, Bangalore, India.

A. Makur is with the School of Electrical and Electronic Engineering, Nanyang Technological University, Singapore.

A. Muthuvel is with Swiss Federal Institute of Technology, Lausanne, Switzerland.

P. V. Reddy is with General Electric, Bangalore, India

Digital Object Identifier 10.1109/TSP.2004.827201 and the remaining filters are designed. In [4], symbolic computation is used to characterize an LPFB of any order. However, none of the above characterizations are complete. In [5], a lattice structure is used to characterize the LPFB so that the analysis polyphase matrix of the FOLPFB becomes $(1 / 2 \sqrt{2})\left[\begin{array}{ll}\left(1+z^{-1}\right) \mathbf{U} & \left(1-z^{-1}\right) \mathbf{U} \\ \left(1-z^{-1}\right) \mathbf{V} & \left(1+z^{-1}\right) \mathbf{V}\end{array}\right]$ $\cdot\left[\begin{array}{cc}\mathbf{U}_{0} & \mathbf{U}_{0} \mathbf{J}_{M / 2} \\ \mathbf{V}_{0} \mathbf{J}_{M / 2} & -\mathbf{V}_{0}\end{array}\right]$, where $\mathbf{U}, \mathbf{V}, \mathbf{U}_{0}$, and $\mathbf{V}_{0}$ are nonsingular $M / 2 \times M / 2$ matrices, and $\mathbf{J}_{k}$ is a $k \times k$ counter identity matrix. It is shown to be complete for FOLPFBs with synthesis length $2 M$ [6].

We briefly describe below the characterization of [1]. Replacing $z^{-1}$ by $\lambda$, the $l$ th-order analysis polyphase matrix $\mathbf{E}(z)$ is seen as a matrix polynomial $\mathbf{E}_{l}(\lambda)$. Any matrix polynomial may be characterized by the Jordan pair (or decomposable pair or spectral data) $(\mathbf{Y}, \mathbf{T}(\lambda)$ ) with $\mathbf{Y}=\left[\begin{array}{ll}\mathcal{X}_{F} & \mathcal{X}_{R}\end{array}\right]$ and $\mathbf{T}(\lambda)=\operatorname{diag}\left(\mathbf{I}_{\Gamma} \lambda-\mathcal{J}_{F}, \mathcal{J}_{R} \lambda-\mathbf{I}_{M l-\Gamma}\right)$, where $\operatorname{diag}()$ represents a block diagonal matrix with the arguments as the blocks in sequence, $\mathbf{I}_{k}$ is the $k \times k$ identity matrix, and $\Gamma$ is the degree of $\left|\mathbf{E}_{l}(\lambda)\right| . \mathcal{X}_{F}$ is the $M \times \Gamma$ canonical set of Jordan chains, and $\mathcal{J}_{F}$ is the $\Gamma \times \Gamma$ Jordan form of $\mathbf{E}_{l}(\lambda)$ (finite Jordan pair or finite spectrum). $\mathcal{J}_{F}$ is block diagonal with Jordan blocks of size $b_{0}, \ldots, b_{n}$ such that $b_{i}$ are nonincreasing positive integers summing up to $\Gamma$. Each Jordan block's diagonal elements are eigenvalues of $\mathbf{E}_{l}(\lambda)$, upper off-diagonal elements are 1 , and the remaining elements are $0 .{ }^{1} \mathcal{X}_{R}$ and $\mathcal{J}_{R}$ are the corresponding $M \times(M l-\Gamma)$ and $(M l-\Gamma) \times(M l-\Gamma)$ matrices of the reversed matrix polynomial $\lambda^{l} \mathbf{E}_{l}\left(\lambda^{-1}\right)$ for the zero eigenvalue (infinite Jordan pair or infinite spectrum). It follows that [7]

$$
\mathbf{E}_{l}(\lambda)=\mathbf{A}\left(\mathbf{I}_{M l}-\mathbf{P}\right) \mathbf{T}(\lambda) \mathbf{S}_{l-1}^{-1} \mathbf{Q}(\lambda)
$$

where

$$
\mathbf{S}_{k}=\left[\begin{array}{cc}
\mathcal{X}_{F} & \mathcal{X}_{R} \mathcal{J}_{R}^{k} \\
\mathcal{X}_{F} \mathcal{J}_{F} & \mathcal{X}_{R} \mathcal{J}_{R}^{k-1} \\
\vdots & \vdots \\
\mathcal{X}_{F} \mathcal{J}_{F}^{k} & \mathcal{X}_{R}
\end{array}\right], \quad \mathbf{Q}(\lambda)=\left[\begin{array}{c}
\mathbf{I}_{M} \\
\lambda \mathbf{I}_{M} \\
\vdots \\
\lambda^{l-1} \mathbf{I}_{M}
\end{array}\right]
$$

$\mathbf{A}$ is an $M \times M l$ matrix such that $\left[\mathbf{S}_{l-2}^{T} \quad \mathbf{A}^{T}\right]^{T}$ is nonsingular, and $\mathbf{P}=\operatorname{diag}\left(\mathbf{I}_{\Gamma}, \mathcal{J}_{R}\right) \mathbf{S}_{l-1}^{-1}\left[\begin{array}{ll}\mathbf{I}_{M l-M} & \mathbf{0}\end{array}\right]^{T} \mathbf{S}_{l-2}$.

For the FIR inverse to exist, $\mathbf{E}_{l}(\lambda)$ has to be a matrix polynomial with a monomial determinant. This is equivalent to all eigenvalues of $\mathbf{E}_{l}(\lambda)$ being zero. Thus, $\mathcal{J}_{F}$ (as also $\mathcal{J}_{R}$ ) should have a zero diagonal. Further, for the first-order $(l=1)$ case, (1) simplifies to the block diagonal characterization [1]

$$
\mathbf{E}_{1}(\lambda)=\mathbf{A T}(\lambda) \mathbf{Y}^{-1}
$$

where $\mathbf{A}$ and $\mathbf{Y}$ are any $M \times M$ nonsingular matrices. Note that $\mathcal{J}_{F}, \mathcal{J}_{R}$ are nilpotent matrices with indices of nilpotency $n_{F}$ and $n_{R}$ ( $n_{F}=b_{0}$, size of the largest Jordan block of $\mathcal{J}_{F}$, etc.). Then, the synthesis polyphase becomes $\mathbf{E}_{1}^{-1}(\lambda)=$ $\mathbf{Y} \operatorname{diag}\left(\mathbf{I}_{\Gamma} \lambda^{-1}+\sum_{i=2}^{n}{ }_{F} \mathcal{J}_{F}^{i-1} \lambda^{-i},-\mathbf{I}_{M-\Gamma}-\sum_{i=1}^{n} R^{-1} \lambda^{i} \mathcal{J}_{R}^{i}\right) \mathbf{A}^{-1}$. It follows that the maximum length of the synthesis filters is $\left(n_{F}+n_{R}\right) M$, and reconstruction delay is $\left(n_{F}+1\right) M-1$. The characterization allows unconstrained parameter optimization and provides control over the length of the synthesis filters and reconstruction delay.

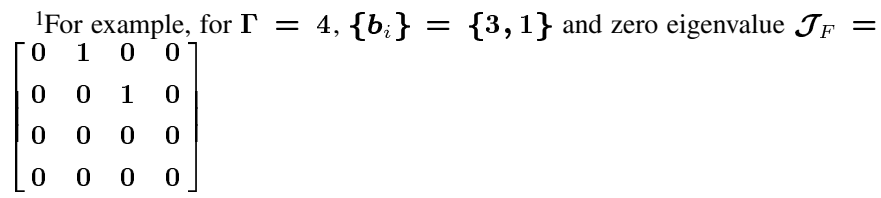

